\title{
On the influence of microscale inertia on dynamic ductile crack extension
}

\author{
N. Jacques ${ }^{1}$, S. Mercier ${ }^{2}$, and A. Molinari ${ }^{2}$ \\ ${ }^{1}$ ENSTA Bretagne, LBMS, 2 rue François Verny, 29806 Brest Cedex 9, France \\ ${ }^{2}$ Université de Lorraine, LEM3, Ile du Saulcy, 57045 Metz Cedex 01, France
}

\begin{abstract}
The present paper is devoted to the modelling of damage by micro-voiding in ductile solids under dynamic loading conditions. Using a dynamic homogenization procedure, a constitutive damage model accounting for inertial effects due to void growth (microscale inertia or micro-inertia) has been developed. The role played by microscale inertia in dynamic ductile crack growth is investigated with the use of the proposed micromechanical modelling. It is found that micro-inertia has a significant influence on the fracture behaviour. Micro-inertia limits the velocity at which cracks propagate. It also contributes to increase the apparent dynamic toughness of the material.
\end{abstract}

\section{Introduction}

The fracture of ductile materials is often the result of the nucleation, growth and coalescence of microscopic voids. In dynamic fracture, micro-voids sustain an extremely rapid expansion which generates strong acceleration of particles in the vicinity of the micro-voids. The role of these microscopic inertial effects in dynamic fracture has been often ignored. For instance, most of the constitutive models for dynamic ductile damage assumed that void expansion is governed by viscous effects, see e.g. $[1,2]$. However, this assumption is in contradiction with several theoretical works dealing with the growth of a single cavity in an unbounded or bounded matrix, which revealed that inertia strongly affects the void evolution $[3,4]$. Several recent works, dedicated to the modelling of spall fracture, indicated that microscale inertia may have a strong influence on the constitutive response of voided ductile media under dynamic loading conditions [5-7].

Studies on the influence of micro-inertia on dynamic ductile crack growth are very seldom. In 1972, Glennie [8] proposed a simple analytical model for void growth in the vicinity of a blunted crack tip in which the effect of microscale inertia is taken into account. Even if Glennie's work relies on some strong assumptions (in particular, the effect of damage due to void growth on the stress state in the vicinity of the crack tip is neglected), it suggested that the inertial resistance to void expansion limits the speed at which cracks can propagate

In the present contribution, a constitutive damage model for porous plastic materials taking microscale inertia into account is proposed. Micro-inertial effects have been incorporated using a dynamic version of Hill-Mandel's homogenization approach, proposed by Molinari and Mercier [9], and are a direct consequence of local material accelerations around the growing voids. Material rate dependence and adiabatic heating due to plastic dissipation are also taken into account. The proposed material model has been implemented in a finite element code. The fracture of an axisymmetric notched bar and a double-edge cracked specimen is investigated. In both cases, the influence of microscale inertia is found to be significant. Because micro-inertia prevents damage to develop too rapidly, a regularizing effect is observed which reduces the mesh sensitivity of the simulations. Micro-inertia is also found to lead to lower crack speed and higher fracture toughness, compared to situation where this contribution is neglected.

\section{Micromechanical modelling of ductile voided solids under dynamic loading conditions}

In the present section, a constitutive damage model for porous ductile solids is introduced. Only the salient features are presented here. For a complete description of the model, the reader should refer to [10].

It is considered that a representative volume element (RVE) can be defined in the porous solid. Micrographic observation of damage in ductile metals generally reveals a wide range of void size. For this reason, the RVE is envisioned as a large ${ }^{a}$ volume of material containing a population of different-sized voids. Moreover, it is assumed that each void is embedded in a spherical shell. This means that, in the present approach, the porous solid is modelled as an aggregate of unit cells, each cell being represented by a hollow sphere (Fig. 1). The relevant micro-inertial effects take place at the unit cell level. Indeed, they are induced by the strong accelerations occurring in the vicinity of the voids during their rapid growth.

A two-step homogenization procedure is adopted to obtain the macroscopic response of the material. Firstly, the response of each individual unit cell is described by using the dynamic hollow sphere model proposed by Molinari and Mercier [9]. This model is based on a special homogenization technique, in which inertial effects caused by internal material motions in the unit cells are taken into account. Secondly, the macroscopic behaviour of the RVE (aggregate of unit cells) is obtained by assuming that all

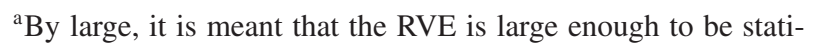
cally representative of the void size distribution in the material.
} 


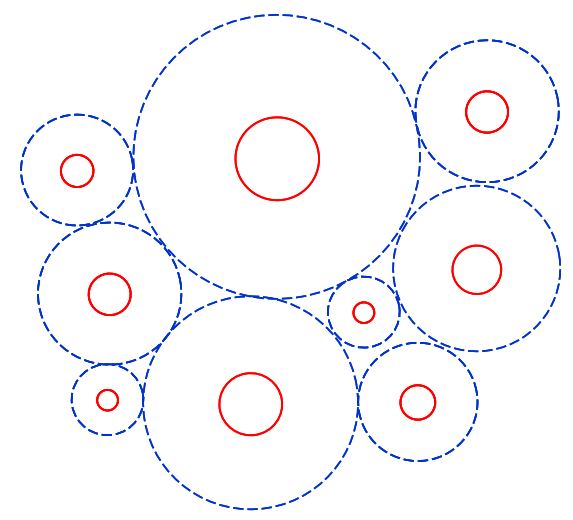

Fig. 1. Representative Volume Element of porous medium.

cells are subjected to the macroscopic strain rate. With this approach, it can be shown that the macroscopic stress $\underline{\tilde{\Sigma}}$ is the sum of two contributions [10]:

$$
\underline{\underline{\Sigma}}=\underline{\underline{\Sigma}}^{s t a}+\tilde{P}^{d y n} \cdot \underline{\underline{I}}
$$

The first term, $\underline{\tilde{\Sigma}}^{s t a}$, is a static stress, related to the constitutive behaviour of the matrix material. The second one, $\tilde{P}^{d y n}$, is a dynamic pressure inherited from material accelerations at the unit cell level. $\tilde{P}^{d y n}$ is given by:

$$
\begin{aligned}
\tilde{P}^{d y n}= & \rho_{0} \tilde{a}^{2}\left[\dot{\tilde{D}}_{m}^{p}\left(\tilde{f}^{-1}-\tilde{f}^{-2 / 3}\right)\right. \\
& \left.+\tilde{D}_{m}^{p^{2}}\left(3 \tilde{f}^{-1}-\frac{5}{2} \tilde{f}^{-2 / 3}-\frac{1}{2} \tilde{f}^{-2}\right)\right]
\end{aligned}
$$

where $\tilde{D}_{m}^{p}$ and $\tilde{f}$ denote respectively the mean component of the macroscopic strain rate tensor and the average porosity in the RVE. The overdot stands for the Lagrangian time derivative. $\tilde{a}$ is an effective void radius, that can be determined from the void size distribution within the material (when all voids have the same size, $\tilde{a}$ is equal to the void radius).

The static stress $\underline{\tilde{\Sigma}}^{\text {sta }}$ can be derived from any existing model of porous ductile solids. In the present work, a viscoplastic version of the standard $\mathrm{GTN}^{\mathrm{b}}$ model is used. The corresponding yield function can be written as:

$$
\Phi=\left(\frac{\tilde{\Sigma}_{e q}^{s t a}}{\bar{\sigma}}\right)+2 q_{1} \tilde{f}_{\cosh }\left(\frac{3}{2} q_{2} \frac{\tilde{\Sigma}_{m}^{s t a}}{\bar{\sigma}}\right)-1-\left(q_{1} \tilde{f}^{2}\right)
$$

where $\tilde{\Sigma}_{e q}^{s t a}$ is the macroscopic effective Mises stress and $\tilde{\Sigma}_{m}^{s t a}$ is the macroscopic static pressure. $\bar{\sigma}$ is the average flow stress of the matrix material. In the present work, the dependence of $\bar{\sigma}$ on strain $\bar{\varepsilon}$, strain-rate $\dot{\bar{\varepsilon}}$ and temperature $T$ is given by the following relationship:

$$
\begin{aligned}
\bar{\sigma}(\bar{\varepsilon}, \dot{\bar{\varepsilon}}, T)= & A\left(\varepsilon_{0}+\bar{\varepsilon}\right)^{n}\left(1+\left(\frac{\dot{\bar{\varepsilon}}}{\dot{\varepsilon}_{0}}\right)^{m}\right) \\
& \times\left(1-\left(\frac{T-T_{r e f}}{T_{m}-T_{r e f}}\right)^{v_{T}}\right)
\end{aligned}
$$

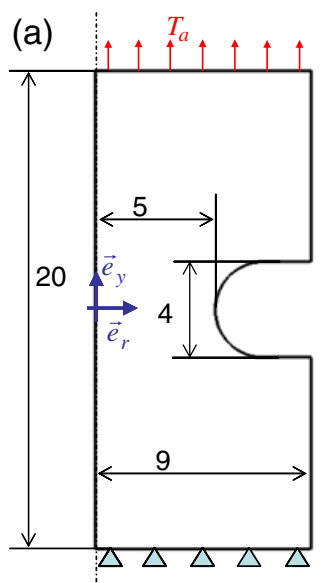

(b)

$T_{a}$

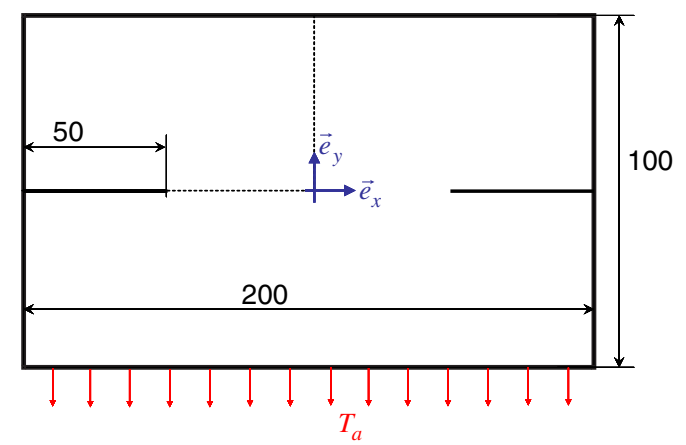

Fig. 2. Geometry of the specimens considered in the present study (dimensions are given in millimetres). (a) axisymmetric notched bar; (b) double-edge cracked plane-strain specimen.

The heating due to plastic dissipation is taken into account by considering adiabatic conditions and assuming that a fixed fraction of plastic work is converted to heat (TaylorQuinney coefficient).

\section{Influence of micro-inertia on dynamic ductile fracture}

The proposed material model has been implemented in the finite element code ABAQUS/Explicit using a usermaterial subroutine. A number of numerical studies of crack propagation have been carried out. Two specimens have been considered: an axisymmetric notched bar (Fig. 2-a) and a precracked plane-strain specimen (Fig. 2-b).

The values of the material parameters used in simulations can be found in [10] and are representative of a medium strength steel. Unless otherwise stated, the initial porosity is taken as $\tilde{f}_{0}=1.5 \times 10^{-4}$. An exponential function is used to describe the initial void size distribution in the material:

$$
w_{0}(a)=\frac{1}{a_{1}} \exp \left(-\frac{a}{a_{1}}\right)
$$

This function has a single parameter, denoted by $a_{1}$, that corresponds to the initial average void radius. Several values for $a_{1}$ ranging between $1.5 \mu \mathrm{m}$ and $5 \mu \mathrm{m}$ have been considered.

\footnotetext{
${ }^{\mathrm{b}}$ Gurson, Tvergaard and Needleman.
} 
a

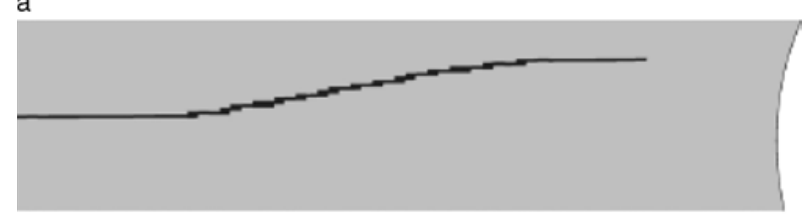

b

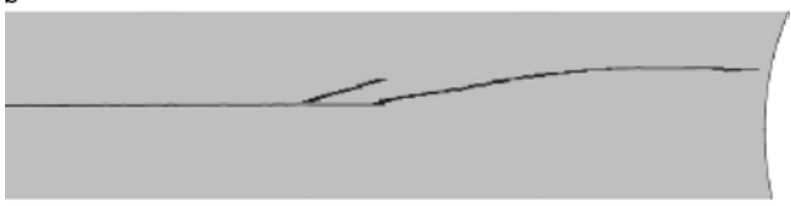

C

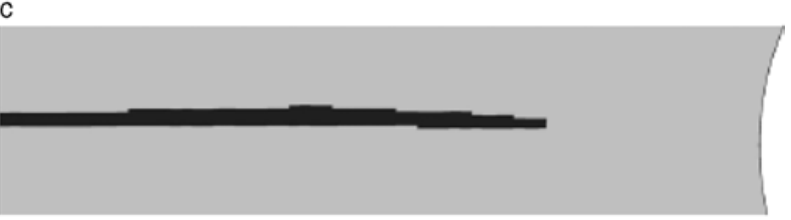

d

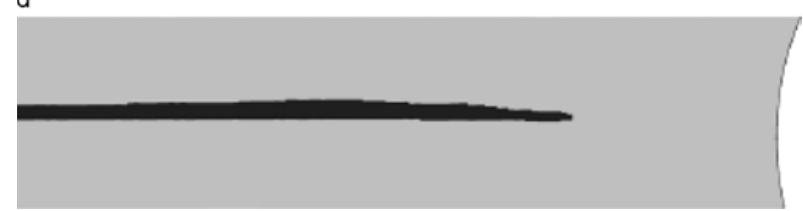

Fig. 3. Crack paths in the axisymmetric notched bar predicted by the micro-inertia based model and GTN one with two different meshes. Elements with porosity greater than 0.3 are painted black. (a) GTN model-coarse mesh; (b) GTN model-fine mesh; (c) present model - coarse mesh; (d) present model - fine mesh. The element size in the gauge region is of $71 \times 22 \mu \mathrm{m}^{2}$ for the coarse mesh and $71 \times 22 \mu \mathrm{m}^{2}$ for the fine mesh.

In order to analyse the role of microscale inertia in dynamic fracture, the results derived from the proposed constitutive framework have been compared to numerical predictions obtained with a viscoplastic version of the standard GTN model, in which micro-inertial effects are neglected. It should be noted that the model with microinertia was built in such a way that it is getting identical to the GTN model when the mean void radius is decreased to zero (as micro-inertia effects become negligible for small void radii), see [10]. A significant influence of micro-inertia was found for the values of $a_{1}$ considered here. In particular, it was observed that accounting for microscale inertia gives rise to a regularizing effect. This means that simulations based on the micro-inertia model exhibit much less mesh dependence than those based on the viscoplastic GTN model. This point is illustrated in Fig. 3, that shows the effect of the mesh size on the crack path in the axisymmetric notched specimen, as predicted by the proposed material model and the GTN one. It should be noted that, in both cases, the crack initiates in the centre of the specimen and propagates in the radial direction to the free surface. With the GTN model (Fig. 3-a and 3b), a cup-cone fracture is observed: at some distance from the centre of the specimen, the crack departs from its initial planar trajectory to form a conical fracture surface. However, the results obtained with the GTN model are clearly mesh-sensitive. Indeed, one sees that the width of the strongly damaged region (plotted in black) depends on the mesh density. In fact, the extent of the damaged

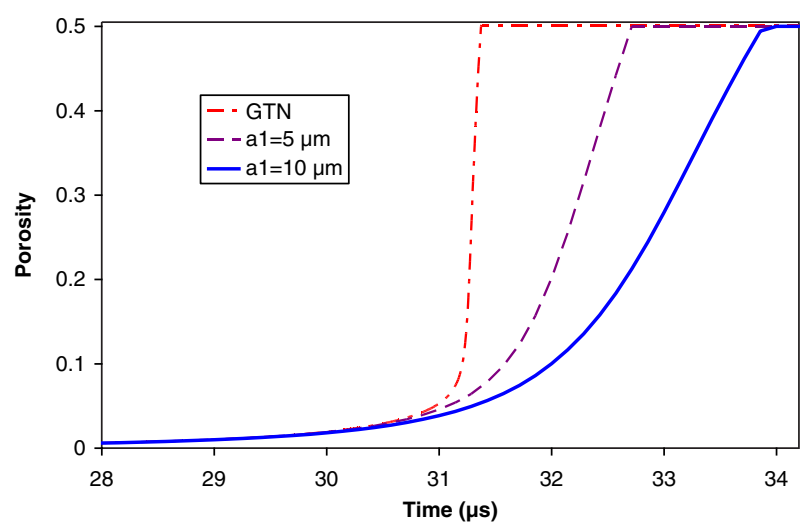

Fig. 4. Time evolution of the porosity in the element at which fracture initiates (this element is inside the highly damaged area represented in Fig. 3, near the axis of symmetry of the specimen). Results obtained with the micro-inertia based model for two values of initial void radius ( $a_{1}=5 \mu \mathrm{m}$ and $\left.10 \mu \mathrm{m}\right)$ are compared to a prediction of the GTN model.

zone is equal to the element size. The distance at which the crack deviates and the amount of crack extension at the considered instant of time are also affected by the mesh density. Figures 3-c and 3-d present the results obtained with the proposed model. It is observed that, in this case, the crack path does not depend on the mesh size. Besides, the width of the region of localized damage is larger and encompasses several elements. The introduction of micro-inertia alleviates the problems of spurious mesh dependence for the present configuration.

In fact, the regularizing effect observed in Fig. 3 is related to the enhanced strain-rate sensitivity of the macroscopic material response due to micro-inertia. Indeed, the dynamic pressure (2) depends on the mean part of the plastic strain rate tensor and on its time derivative. This means that accounting for microscale inertia gives rise to an additional strain rate sensitivity at the scale of the RVE (additional with respect to the one related to the viscoplastic behaviour of the matrix material).

In the present case, the onset of fracture (near the centre of the specimen) and its subsequent propagation is associated to a strain localization mechanism. At some stage of the deformation process, damage and deformation tend to concentrate in a narrow band (this band corresponds to the region painted black in Fig. 3). It is obvious that the appearance of a narrow band of intense straining implies a strong increase of the strain rate in this band. As mentioned previously, the dynamic pressure depends on the time derivative of the plastic strain rate. For this reason, micro-inertia prevents the strain rate to increase too rapidly in the narrow zone of localized deformation. Therefore, the localization process occurs in a more progressive way when microscale inertia is taken into account. At a given instant of time, the localization band is larger with the micro-inertia based model than with the GTN one. This explains the reduced mesh sensitivity observed in Fig. 3.

The stabilizing effect of micro-inertia on the damage accumulation is illustrated by Fig. 4, that presents the time evolution of the porosity in the element where fracture initiates (this element is obviously inside the band of 


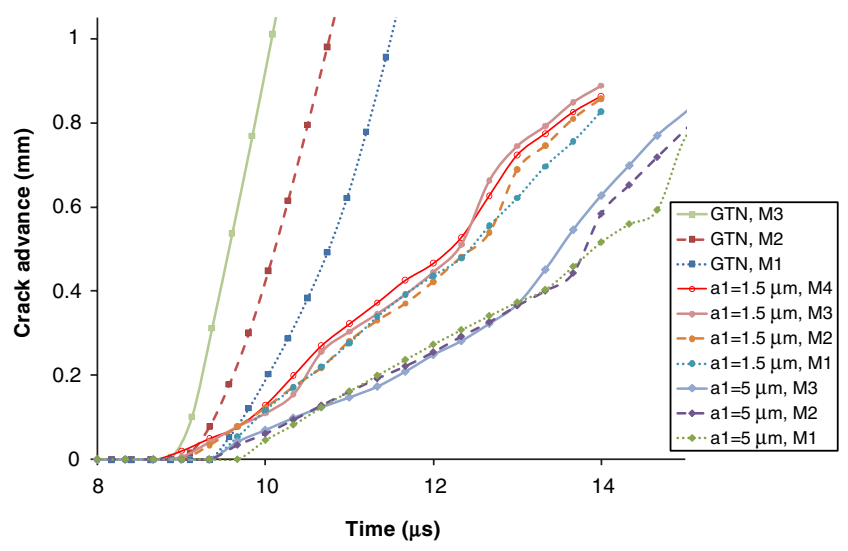

Fig. 5. Crack growth behaviour predicted by the present model (with $a_{1}=1.5 \mu \mathrm{m}$ and $a_{1}=5 \mu \mathrm{m}$ ) and the GTN one for several levels of mesh refinement. Element size in the near crack tip region: M1: $20 \times 20 \mu \mathrm{m}^{2}$, M2: $10 \times 10 \mu \mathrm{m}^{2}, \mathrm{M} 3: 7.25 \times 5 \mu \mathrm{m}^{2}$, M4: $3.625 \times 2.5 \mu \mathrm{m}^{2}$.

localized deformation). Results obtained with the GTN model and the present one for several values of initial void radius ( $a_{1}=5 \mu \mathrm{m}$ and $\left.a_{1}=10 \mu \mathrm{m}\right)$ are compared. It is worth noting that micro-dynamic effects become more pronounced for larger values of initial void radius, see Eq. (2). With the GTN model, there is an explosive increase in porosity at the time corresponding to the occurrence of strain localization. A more gradual evolution of the porosity is observed with the micro-inertia based model. Besides, the stabilizing effect of micro-inertia is enhanced when the initial void radius increases. In fact, the responses observed in Fig. 4 are related to the occurrence of a cavitation instability [11]. For the considered loading conditions, at one point, the material is subjected to stresses greater than its static stress-carrying capability. Subsequently, void growth is unstable and dominated by inertia effects. Hence, if dynamic effects due to void expansion are neglected, an excessively fast porosity accumulation is predicted, leading to a pathological strain localization.

From other simulations, presented in [10], it has been observed that the size of the area of localized deformation is linked to the initial void distribution. For a given value of the initial porosity, the extent of the damage region is almost proportional to the average distance between voids (or equivalently to the initial void radius). This means that the present constitutive framework is able to capture size effects arising from the microstructure of the material. Under quasi-static conditions, the description of microstructural length-scale effects requires the use of non-local models, in which the constitutive response of a material point depends on the deformation in its neighbourhood [12]. The present constitutive model is a standard (local) one. However, by taking into account micro-inertia, the proposed model can capture size effects related to the characteristic length scale of the material microstructure when dynamic loadings are considered (obviously, the effect of micro-inertia will vanish under quasistatic loading).

Let us now consider the double-edge cracked specimen (Fig. 2-b). Figure 5 presents crack advance versus time

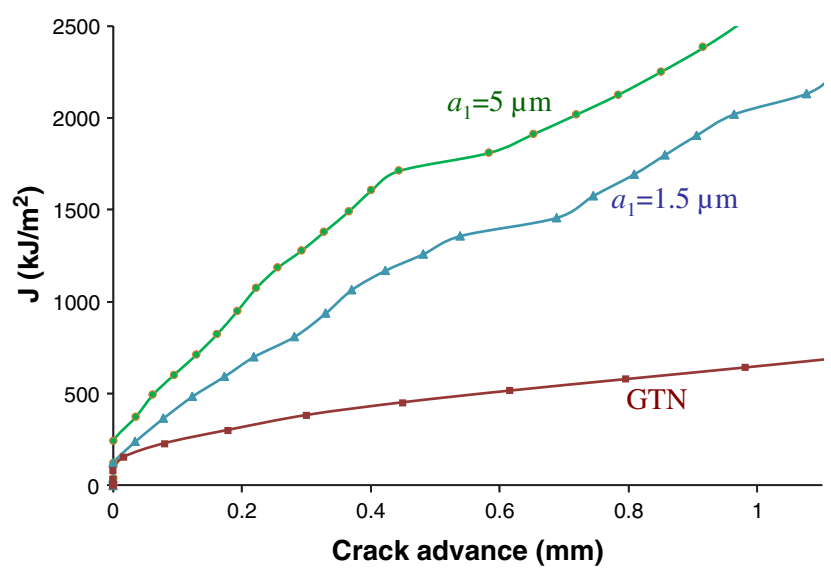

Fig. 6. $J$-resistance curves for the double-edge cracked specimen derived from the micro-inertia based model (with $a_{1}=1.5 \mu \mathrm{m}$ and $\left.a_{1}=5 \mu \mathrm{m}\right)$ and the GTN one.

curves obtained with the present damage model for two values of initial mean void radius $\left(a_{1}=1.5 \mu \mathrm{m}\right.$ and $a_{1}=$ $5 \mu \mathrm{m})$ and with the GTN model. Several levels of mesh refinement are considered. It is observed that micro-inertia has a significant influence on the crack growth behaviour. With the GTN model, the average crack speed over the first millimetre of crack growth ranges between 580 and $970 \mathrm{~m} / \mathrm{s}$, depending on the mesh size. With the present model, cracks propagate at about $180 \mathrm{~m} / \mathrm{s}$ for $a_{1}=1.5 \mu \mathrm{m}$ and at $140 \mathrm{~m} / \mathrm{s}$ for $a_{1}=5 \mu \mathrm{m}$. Microscale inertia limits the velocity at which cracks propagate. Figure 5 also shows that the simulations based on the present model exhibit much less mesh sensitivity that those using the GTN model. The crack growth behaviour predicted by the micro-inertia based model is almost mesh-independent, provided that the element size is small enough to resolve the damage distribution in the process zone.

In order to characterize the influence of microscale inertia on the dynamic toughness of the material, the $J$ integral have been computed. Figure 6 presents $J$-resistance curves for ductile crack growth computed with the present model (for $a_{1}=1.5 \mu \mathrm{m}$ and $a_{1}=5 \mu \mathrm{m}$ ) and with the GTN model. A significant effect of micro-inertia on the fracture resistance is observed. For a given amount of crack growth, the value of $J$ obtained with the proposed constitutive model is greater than the one derived from the GTN model. Moreover, $J$ increases with the initial mean void radius. Several experimental studies have revealed that the dynamic toughness of a material is generally larger then its quasi-static counterpart $[13,14]$. The results presented in Fig. 6 suggest that microscale inertia may be in part responsible for this phenomenon.

Figure 7 presents the effect of the initial porosity on the crack advance versus time and $J$-resistance curves. The reference configuration is defined by $\tilde{f}_{0}=1.5 \times$ $10^{-4}$ and $a_{1}=5 \mu \mathrm{m}$. It should be noted that the initial porosity is related to the number of voids per unit volume $N_{0}$ and the initial mean void radius $a_{1}$ by the following relationship [10]:

$$
\tilde{f}_{0}=8 \pi N_{0} a_{1}^{3}
$$



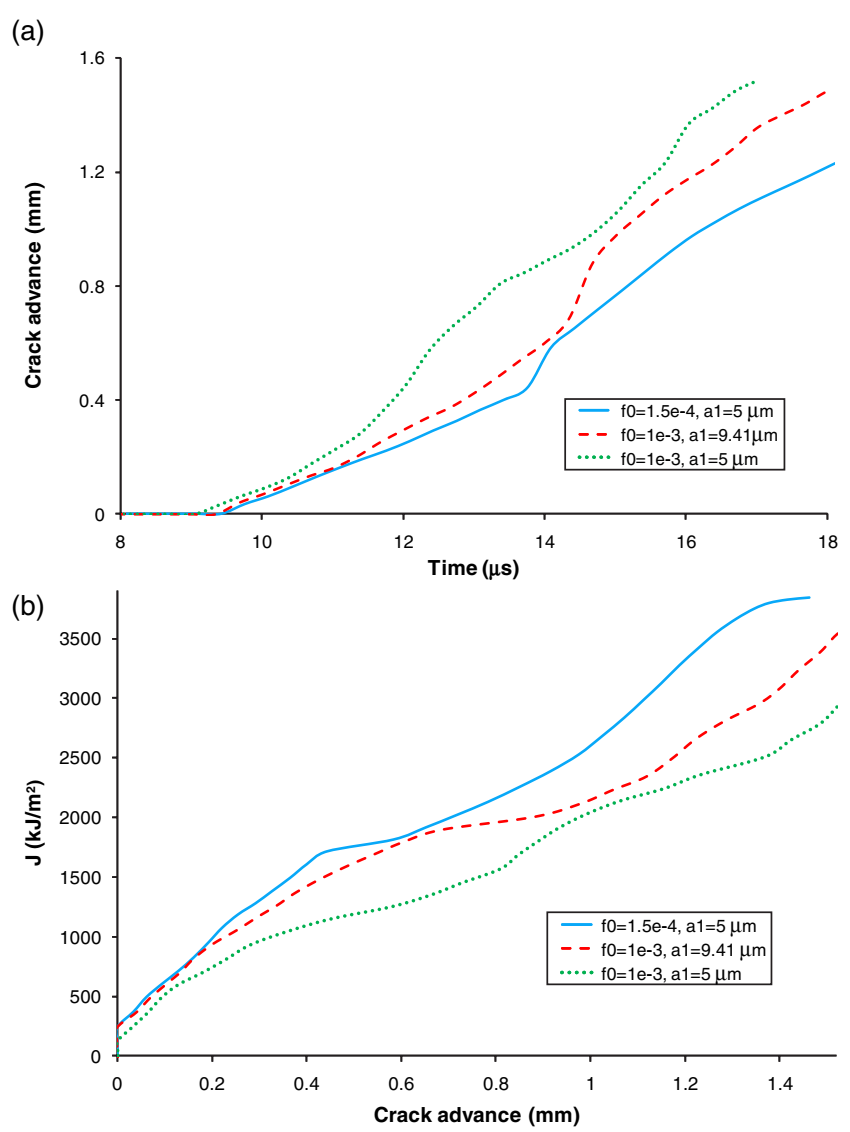

Fig. 7. Effect of the initial porosity on (a) the time evolution of the crack length and (b) the $J$-resistance curve for the doubleedge cracked specimen.

Thus, a larger initial porosity can be obtained (i) by increasing the number of void per unit volume (here, $N_{0}$ is increased by a factor of 6.667, the corresponding configuration is defined by $\tilde{f}_{0}=1 \times 10^{-3}$ and $\left.a_{1}=5 \mu \mathrm{m}\right)$ and (ii) by increasing the value of the initial mean void radius $\left(\tilde{f}_{0}=1 \times 10^{-3}\right.$ and $\left.a_{1}=9.41 \mu \mathrm{m}\right)$. Figure 7 shows that, in both cases, the increase in porosity gives a higher crack speed and a smaller fracture resistance. Nevertheless, the way the initial porosity is changed matters. Larger crack speed and lower fracture toughness are obtained when the increase of the initial porosity is achieved by increasing the number of voids per unit volume, as compared to the case where the porosity increase is obtained by considering a larger mean void radius. This is due to the fact that, for the same porosity, the effect of micro-inertia is more pronounced in a configuration with a smaller number of large voids than with a larger number of small voids.

\section{Summary and conclusions}

A constitutive model for damage in ductile materials under dynamic conditions has been developed. The original feature of this model is to take microscale inertia into account. Micro-dynamic effects have been incorporated in the formulation by using a dynamic homogenization method and are a direct consequence of the acceleration sustained by the matrix material near the expanding voids.

The proposed model has been used to analyse dynamic (unstable) crack growth in an axisymmetric notched bar and a double-edge cracked specimen. In both cases, the role of micro-inertia was found to be significant. In particular, micro-inertia plays an important role in strain localization phenomena. As micro-inertia impedes void growth in the narrow region where strain tends to concentrate, the resulting damage accumulation occurs in a more progressive manner. For this reason, simulations based on the present modelling show much less mesh sensitivity than those based on a viscoplastic version of the Gurson-TvergaardNeedleman model. Micro-inertia provides a clear regularizing effect, which is related to microstructural parameters (initial void size distribution and porosity). The proposed model is able to capture size effects arising from the microstructure of the material.

Micro-inertia is also found to have a strong influence on crack speed and fracture toughness.

\section{References}

1. L. Seaman, D.R. Curran, D.A. Shockey, J. Appl. Phys. 47, 4814-4826 (1976)

2. A. Needleman, V. Tvergaard, Int. J. Fract. 49, 41-67 (1991)

3. M. Ortiz, A. Molinari, J. Appl. Mech. 59, 48-53 (1992)

4. W. Tong, G. Ravichandran, J. Appl. Mech. 62, 633639 (1995)

5. C. Czarnota, N. Jacques, S. Mercier, A. Molinari, J. Mech. Phys. Solids 56, 1624-1650 (2008)

6. T. Wright, K.T Ramesh, J. Mech. Phys. Solids 56, 336-359 (2008)

7. N. Jacques, S. Mercier, A. Molinari, Int. J. Fract. 162, 159-175 (2010)

8. E.B. Glennie, J. Mech. Phys. Solids 20, 415-429 (1972)

9. A. Molinari, S. Mercier, J. Mech. Phys. Solids 49, 1497-1516 (2001)

10. N. Jacques, S. Mercier, A. Molinari, J. Mech. Phys. Solids (in press). doi:10.1016/j.jmps.2011.12. 010

11. Y. Huang, J.W. Hutchinson, V. Tvergaard, J. Mech. Phys. Solids 39, 223-241 (1991).

12. J.-B. Leblond, G. Perrin, J. Devaux, J. Appl. Mech. 61, 236-242 (1994).

13. T. Kanazawa, S., Machida, T., Teramoto, H., Yoshinari, Exp. Mech. 21, 78-88 (1981).

14. A.J. Rosakis, A.T., Zehnder, Int. J. Fract. 27, 169-186 (1985). 